\title{
Skew Hadamard designs and their codes
}

\author{
Jon-Lark Kim • Patrick Solé
}

Received: 30 May 2007 / Revised: 9 October 2007 / Accepted: 15 October 2007 /

Published online: 6 March 2008

(C) The Author(s) 2008

\begin{abstract}
Skew Hadamard designs $(4 n-1,2 n-1, n-1)$ are associated to order $4 n$ skew Hadamard matrices in the natural way. We study the codes spanned by their incidence matrices $A$ and by $I+A$ and show that they are self-dual after extension (resp. extension and augmentation) over fields of characteristic dividing $n$. Quadratic Residues codes are obtained in the case of the Paley matrix. Results on the $p$-rank of skew Hadamard designs are rederived in that way. Codes from skew Hadamard designs are classified. An optimal self-dual code over $G F(5)$ is rediscovered in length 20 . Six new inequivalent $[56,28,16]$ self-dual codes over $G F(7)$ are obtained from skew Hadamard matrices of order 56, improving the only known quadratic double circulant code of length 56 over $G F(7)$.
\end{abstract}

Keywords Skew Hadamard designs $\cdot$ Self-dual codes

AMS Classifications $05 \mathrm{~B} 20 \cdot 94 \mathrm{~B} 25$

\section{Introduction}

In $[1,2]$ a systematic study of the codes of the designs of Hadamard matrices was undertaken. With a Hadamard matrix $H$ of order $4 n$ can be attached a 3-design $\mathcal{T}$ of parameters $3-(4 n, 2 n, n-1)$. The code $C_{p}(\mathcal{T})$ (row space of the block versus points incidence matrix of $\mathcal{T}$ over a finite field) is self-orthogonal [1]. If, furthermore, $H$ is skew Hadamard (or SH), then $C_{p}(\mathcal{T})$ is self-dual [11].

In this article we give an independent coding theoretic proof of the latter result. The derived design $\mathbf{D}$ of $\mathcal{T}$ has parameters $2-(4 n-1,2 n-1, n-1)$. We study the codes spanned by

J.-L. Kim

Department of Mathematics, University of Louisville, Louisville, KY 40292, USA

e-mail: jl.kim@louisville.edu

P. Solé $(\bowtie)$

CNRS, I3S, Les Algorithmes - bt. Euclide B, BP.121, 2000 route des Lucioles, 06903 Sophia Antipolis

Cedex, France

e-mail: sole@unice.fr 
the incidence matrices of $\mathbf{D}$ and of its complement and show that they are self dual after extension (resp. extension and augmentation) over fields of characteristic dividing $n$. Quadratic Residues codes are obtained in the case of the Paley matrix. We classify self-dual codes from skew Hadamard matrices of order $4 n(2 \leq n \leq 7)$ and enumerate self-dual codes from skew Hadamard matrices of order $4 n(8 \leq n \leq 15, n=18,21)$. In particular, an optimal self-dual code over $G F(5)$ is constructed in length 20 , rediscovering the work of [19]. We also find six new inequivalent $[56,28,16]$ self-dual codes over $G F(7)$ from skew Hadamard matrices of order 56 . These are inequivalent to the only known quadratic double circulant code of length 56 over $G F(7)$ [3].

\section{Skew Hadamard designs}

Throughout the paper we denote by $I$ (resp. $J$ ) the identity (resp. all-one) matrix of a suitable order. A Hadamard matrix $H$ is said to be skew if it is of the form $I+K$ with $K$ a skew symmetric zero diagonal matrix. Such a matrix induces a 3 -design $\mathcal{T}$ of parameters 3 $(4 n, 2 n, n-1)$, whose derived design $\mathbf{D}$ has parameters $2-(4 n-1,2 n-1, n-1)$. By a skew Hadamard design we will mean such a design throughout. Let $A$ be the block versus points $\{0,1\}$ valued incidence matrix of a skew Hadamard design of parameters $(4 n-1,2 n-1, n-1)$ over a finite field $F$ of characteristic $p$. By definition it is a matrix $A$ of order $4 n-1$ satisfying

$$
A A^{T}=n I+(n-1) J
$$

and

$$
A J=J A=(2 n-1) J .
$$

The skewness property translates into $A$ being the adjacency of a tournament digraph, that is,

$$
A+A^{T}+I=J .
$$

In all the article, we assume that $p$ divides $n$. As a consequence

$$
A A^{T}=-J \text {. }
$$

The following result is proved in [11] building on Sylvester's law of nullity for a matrix product [12, Theorem 5.6.2, Theorem 5.6.5].

Proposition 1 (T. S. Michael) The p-rank of A (resp. J - A) is $2 n($ resp. $2 n-1)$.

We give an independent proof based on coding theory. Let $C(A)=\left\langle A^{+}\right\rangle$denote the $F$-span of $A^{+}$, which is $A$ extended by an all-one column. In the notations of [2] this is the code $C_{p}(\mathcal{T})$ of the 3-design $\mathcal{T}$. Let $D(A)$ denote $\left\langle(I+A)^{-}\right\rangle$augmented by adding the all-one vector to its generating set. Here $M^{-}$denotes $M$ extended by an all-zero column. By $r k(M)$ (resp. $\operatorname{cork}(M)$ ) we denote the rank (resp. corank i.e., the dimension of its kernel) of $M$ over $F$. The following result implies the preceding proposition.

Proposition 2 The codes $C(A)$ and $D(A)$ are self-dual over $F$.

Proof First, we observe that $\langle I+A\rangle$ is self-orthogonal by computing, using Eqs. 1 and 3, the product $(A+I)(A+I)^{T}=A A^{T}+J=O$. Hence $r k(A+I) \leq 2 n-1$. By a similar argument $C(A)$ is self-orthogonal and $r k(A) \leq 2 n$. Adding up these two bounds we obtain

$$
r k(A+I)+r k(A) \leq 4 n-1 .
$$


Next, the eigenspaces of $A$ with respect to 0 and -1 are disjoint, and this entails that their dimensions add up to at most the ambient space dimension

$$
\operatorname{cork}(A+I)+\operatorname{cork}(A) \leq 4 n-1,
$$

or, equivalently

$$
r k(A+I)+r k(A) \geq 4 n-1 .
$$

Equations 4 and 5 together imply that equality holds in all preceding inequalities.

Note that in general for an arbitrary Hadamard matrix one has only an upper bound on the $p$-rank [2, Theorem 7.4.1]. The next result is well-known for QR codes over $G F(2)$ of length a multiple of 8 .

Corollary 1 When $F=G F(2)$ the codes $C(A)$ and $D(A)$ are Type II. Further $\left\langle I+A^{T}\right\rangle$ is the even part of $\langle A\rangle$. In fact $\langle A\rangle=\left\langle I+A^{T}\right\rangle \oplus \mathbf{1}$.

Proof The first statement is immediate by the fact that all rows of $A$ have weight $2 n-1$. The second statement comes from the relation

$$
A\left(I+A^{T}\right)^{T}=A(J-A)^{T}=A\left(J-A^{T}\right)=n J=O,
$$

a direct consequence of Eqs. 2 and 1. This implies that $\left\langle I+A^{T}\right\rangle \subseteq\langle A\rangle^{\perp}$. This bound is an equality by dimension count. Hence $\left\langle I+A^{T}\right\rangle^{\perp}=\langle A\rangle$. The code $\left\langle I+A^{T}\right\rangle$ is even, being self-orthogonal. The result follows.

\section{Extended QR codes from Paley Hadamard matrices}

We recall a definition of a Quadratic Residue code (QR code) of prime length $l$ over $G F(p)$, where $p$ is another prime which is a quadratic residue $\bmod l$ (here we interchange $p$ and $l$ in [10, Chap. 16]). Let $Q$ be the set of quadratic residues modulo $l$, and $N$ the set of nonresidues modulo $l$. The set $Q$ is closed under multiplication by $p$ as $p \in Q$. Let $\alpha$ be a primitive $l$ th root of unity in some extension of $G F(p)$. Let $q(x)=\prod_{r \in Q}\left(x-\alpha^{r}\right)$. Define the $Q R$ code $\mathcal{Q}$ to be the cyclic code of length $l$ over $G F(p)$ with generator polynomial $q(x)$. Define $\theta:=\sum_{i=1}^{l-1}\left(\frac{i}{l}\right) \alpha^{i}$ to be the Gaussian sum, where $\alpha$ is a primitive $l$ th root of unity in some extension of $G F(p)$ and $\left(\frac{i}{l}\right)$ is the Legendre symbol. Note that $\theta \in G F(p)$. Further the following is known [10, Chap. 16].

Lemma 1 If $l \equiv-1(\bmod 4)$, then $\theta^{2}=-l$.

For a precise definition of Paley Hadamard matrices (hereby abbreviated as $\mathrm{PH}$ ) we refer to [10, pp. 47-48]. With the above notation, let it suffice to say they are constructed as in [10,p. 48] from the Jacobsthal matrix $\mathcal{J}$ with typical entry $\mathcal{J}_{x, y}=\left(\frac{y-x}{l}\right)$.

Proposition 3 Let $l:=4 n-1$ be a prime and $p$ be a prime dividing $n$ such that $p$ is a quadratic residue mod l. Suppose $H$ is the Paley Hadamard matrix of order $4 n$. Let $A$ be the associated incidence matrix from $H$. Then $C(A)$ is the extended quadratic residue code $\hat{\mathcal{Q}}$ over $G F(p)$.

Proof First we calculate the idempotent of the quadratic residue code $\mathcal{Q}$ of a prime length $l$ over $G F(p)$ in terms of residues and nonresidues mod $l$. In fact, the idempotent of the 
quadratic residue code $\mathcal{Q}$ of a prime length $l$ over $G F(p)$ is given in [10, Theorem 4, Chap. 16] as follows.

$$
E_{q}(x)=\frac{1}{2}\left(1+\frac{1}{l}\right)+\frac{1}{2}\left(\frac{1}{l}-\frac{1}{\theta}\right) \sum_{r \in Q} x^{r}+\frac{1}{2}\left(\frac{1}{l}+\frac{1}{\theta}\right) \sum_{n \in N} x^{n} .
$$

Here $\theta$ is the Gaussian sum given above. Then $\theta$ satisfies $\theta^{2}=-l$ by Lemma 1 . As $l \equiv-1$ $(\bmod p)$, we get $\theta^{2}=1(\bmod p)$, so $\theta= \pm 1$. If $\theta=-1$ then we choose the primitive element $\alpha$ so that $\theta=1$ (for example, let $\beta:=\alpha^{c}$ where $\left(\frac{c}{l}\right)=-1$. Then the Gaussian sum based on $\beta$ becomes 1). Hence $E_{q}(x)=-\sum_{r \in Q} x^{r}$. It is not difficult to check that the extended code $\hat{\mathcal{Q}}$ is the same as $C(A)$.

A duadic code is a class of cyclic codes generalizing quadratic residue codes [7]. A multiplier $\mu_{a}$ of $\mathbb{F}_{p}[x] /\left(x^{n}-1\right)$ is a ring automorphism induced by $x \mapsto x^{a}$. A cyclotomic coset is an orbit of $\mu_{p}$ on $\mathbb{Z}_{n}$, where we assume $n$ and $p$ to be coprime. A splitting is a partition of $\mathbb{Z}_{n} \backslash 0$ into two unions of cyclotomic cosets $U_{1}$ and $U_{2}$ swapped by a multiplier. Recall that the zeros of a cyclic code of length $n$ over a field $F$ are the zeros of its generator polynomial [10, p. 199], and its characteristic set $T$ is the set of exponents of its zeros $Z=\left\{\alpha^{t} \mid t \in T\right\}$, where $\alpha$ is a primitive root of order $n$ in the algebraic closure of $F$. The four duadic codes attached to a splitting are defined by their characteristic sets $U_{1}, U_{2}$ (odd-like case) or $U_{1}+0, U_{2}+0$ (even like case). Extension of odd like duadic codes are self dual when $a=-1$. Quadratic Residue codes above correspond to $U_{1}$ the set of quadratic residues of $\mathbb{Z}_{n}$, for $n$ prime.

In [13] Pless related binary duadic codes to cyclic even tournaments. The adjacency matrix $A$ of a tournament digraph is called cyclic [13] if each row of $A$ is a cyclic shift of the previous row and even if $A A^{T} \equiv I(\bmod 2)$.

We note that our matrix $A$ is not even as $A A^{T} \equiv-J(\bmod p)$. But if we assume that $A$ is cyclic, then we obtain duadic codes over $G F(p)$ from $A$ as follows.

Lemma 2 [7, Theorem 6.4.1] Let $C$ be any $[n,(n-1) / 2]$ cyclic code over $G F(q)$, where $q$ is a power of a prime $p$. Then $C$ is self-orthogonal if and only if $C$ is an even-like duadic code whose splitting is given by $\mu_{-1}$.

Proposition 4 Suppose that $A$ is the cyclic incidence matrix of a skew Hadamard design of parameters $(4 n-1,2 n-1, n-1)$. Let $C_{1}:=\langle I+A\rangle$ be the code over a finite field $F$ of characteristic $p$ generated by the rows of $I+A$ and let $C_{2}:=\left\langle I+A^{T}\right\rangle$. Similarly let $D_{1}:=\left\langle A^{T}\right\rangle$ and $D_{2}:=\langle A\rangle$. Then the following hold.

1. $C_{i}(i=1,2)$ is an even-like duadic code over $F$ whose splitting is given by $\mu_{-1}$.

2. $D_{i}$ is the odd-like duadic code of $C_{i}(i=1,2)$ whose splitting is given by $\mu_{-1}$.

Proof We have shown in the proof of Proposition 2 that $C_{1}$ (similarly $C_{2}$ ) is self-orthogonal over $F$ with dimension $2 n-1$. Hence the first statement follows from Lemma 2. Following the proof of Corollary 1 , we see that $C_{i}$ is a codimension one subcode of $D_{i}(i=1,2)$. As 1 is not in $C_{i}(i=1,2), D_{i}$ is the odd-like duadic code of $C_{i}(i=1,2)$.

Applying the square root bound of duadic codes (cf. [7, Theorem 6.5.2]), we get the square root bound of duadic codes from the cyclic incidence matrix of a skew Hadamard design of parameters $(4 n-1,2 n-1, n-1)$.

Corollary 2 (Square Root Bound) Let $D_{i}(i=1,2)$ of length $4 n-1$ be as above. Let $d_{0}$ be their (common) minimum odd-like weight. Then the following hold. 
1. $d_{0}^{2}-d_{0}+1 \geq 4 n-1$.

2. Suppose $d_{0}^{2}-d_{0}+1=4 n-1$ where $d_{0}>2$, then for $i=1,2$

(a) $d_{0}$ is the minimum weight of $D_{i}$.

(b) the supports of the minimum weight codewords of $D_{i}$ form a cyclic projective plane of order $d_{0}-1$.

(c) the minimum weight codewords of $D_{i}$ are multiples of binary vectors.

(d) there are exactly $(4 n-1)(p-1)$ minimum weight codewords in $D_{i}$.

Generalizations of the last two results to the case when $\mathbf{D}$ is an Abelian difference set are in [15].

\section{Their codes}

In this section, we classify or enumerate self-dual codes from SH matrices of reasonable sizes. In the following, we do not mention the case when $H$ is the Paley Hadamard matrix, as this leads to quadratic residue codes. Recall that a self dual code is Type II if it is binary with all weights multiple of 4 and Type III if it is ternary.

$4.1 n=2$ or 3

There is a unique SH matrix of order 8 [9], whose $C(A)$ is the binary Hamming $[8,4,4]$ code. Similarly there is a unique SH matrix of order 12 [9], whose $C(A)$ is the ternary Golay $[12,6,6]$ code. These can be explained from Proposition 3.

$4.2 n=4$

It is well known that there are two SH matrices of order 16 . These matrices can be constructed from the adjacency matrix $A$ of the unique 2-class association scheme of order 15 [5] and its transpose $A^{T}$. We construct two inequivalent extremal Type II [16, 8, 4] codes of length 16 from $C(A)$ and $C\left(A^{T}\right)$. As there exist only two such codes, we have shown that every extremal Type II code of length 16 can be obtained from a SH matrix of order 16.

\section{$4.3 n=5$}

It is known that there are exactly two SH matrices, one being PH. Again these matrices can be obtained from the two 2-class Association schemes [5]. We construct two inequivalent optimal [20, 10, 8] self-dual codes over $G F(5)$. More precisely, No. 2 of [5] is not of Paley type and gives an optimal [20,10,8] self-dual code $S H_{20}$ over $G F(5)$ by construction $C(A)$, rediscovering the work of [19]. Its order of the automorphism group is $2^{9} \cdot 3 \cdot 5$. In [4], only two self-dual [20,10,8] codes over $G F(5)$, denoted by $Q D C_{20}$ and $X Q_{19}$, are given, where their group orders are $2^{8} \cdot 3^{2} \cdot 5$ and $2^{4} \cdot 3^{2} \cdot 5 \cdot 19$, respectively. We recall that there are three inequivalent Hadamard matrices of order 20 [17]. We have checked that the second and the third Hadamard matrices in [17] produce $S H_{20}$ and $X Q_{19}$, respectively while the first Hadamard matrix in [17] produce $Q D C_{20}$. Therefore we have shown the following.

Proposition 5 There exist at least three optimal [20, 10,8] self-dual codes over GF(5), all of which are from Hadamard matrices of order 20, two being from skew Hadamard matrices of order 20.

For more detail about these codes, see [19, Table 1, Remark 3]. 
Table 1 Type II codes from the 16 skew Hadamard matrices of order 24

\begin{tabular}{ll}
\hline Codes [14] & Skew Hadamard matrices [18] \\
\hline$F_{24}$ & $\{1,3,11,12\}$ \\
$D_{24}$ & $\{2,4,7,8,13\}$ \\
$C_{24}$ & $\{5,9,10\}$ \\
$A_{24}$ & $\{6\}$ \\
$E_{24}$ & $\{15\}$ \\
$G_{24}$ & $\{14,16\}$ \\
\hline
\end{tabular}

\section{$4.4 n=6$}

There are (up to equivalence) $16 \mathrm{SH}$ matrices, one being $\mathrm{PH}$. We use the classification given by Spence [18]. Binary and ternary codes of length 24 are obtained as follows. Assmus and Key [1] described in detail the binary and ternary codes from Hadamard matrices of order 24, but they did not consider which codes are from skew Hadamard matrices. We have checked that there are exactly six Type II codes from the $16 \mathrm{SH}$ matrices, one of them is the extended Golay code $G_{24}$ of length 24 and the other have minimum weight 4 . For detail, see Table 1. Here the first column refers to the binary Type II codes from [14] and the second column refers to the indices of the skew Hadamard matrices in [18].

Further the $16 \mathrm{SH}$ matrices produce exactly 9 Type III codes of length 24 . Two such codes are the extended QR code of length 24 and the symmetry code of length 24 .

\section{$4.5 n=7$}

The 65 skew Hadamard matrices of order 28 in [18, pp. 239-243] reduce to 54 inequivalent SH matrices, one being PH [18]. For example, the SH matrix with No. 11 in [18] is equivalent to the SH matrix with No. 7in [18] since both come from the Hadamard matrix with No. 233 in [18, p. 217].

We consider codes over $G F(7)$ of length 28. Each matrix using the construction $C(A)$ produces a self-dual $[28,14,9]$ code over $G F(7)$ and the 54 codes obtained this way are all inequivalent as one might expect. In Table 2, we describe the orders of the permutation automorphism groups of the 54 codes. It is interesting to compare the orders of the SH matrices with those of the corresponding codes. For example, the orders of the automorphism groups of the SH matrices with No. 1 and 2 [18] are 2 and 1 respectively while the group orders of the corresponding codes are 12 and 6 respectively. In most cases, the group order of the code is $6 \times$ (the group order of the SH matrix). We do not have any explanation for this fact.

We note that these codes have minimum weight one less than the best known $[28,14,10]$ codes [4].

For more general results in the non-SH case, see [19].

\section{$4.6 n=8$}

There are $\geq 6$ SH matrices, one being PH [16]. We only get binary Type II codes of length 32 by Corollary 1. It is known [7] that there are exactly two binary extended duadic self-dual codes of length 32, one of which is the extended binary QR code of length 32, and the other the Reed-Muller code of order 2. Both are extremal. The QR code is constructed from the PH matrix of order 32 by Proposition 4. We leave as an open problem to know if the remaining codes are extremal. 
Table 2 Orders of the permutation automorphism groups of self-dual $[28,14,9]$ codes over $G F(7)$ from the 54 $\mathrm{SH}$ matrices of order 28

\begin{tabular}{ll}
\hline $\mid$ PAut $(\mathrm{C}) \mid$ & Skew Hadamard matrices $[18]$ \\
\hline 6 & $\{2,7,8,9,16\}$ \\
12 & $\{1,3,4,6,17,29,30,31,34,37\}$ \\
18 & $\{5,12,23,33,42,45,46,47,51,53\}$ \\
24 & $\{14,44\}$ \\
36 & $\{13,19,24,26,28,35,38,40,43\}$ \\
48 & $\{10,20\}$ \\
54 & $\{41\}$ \\
72 & $\{21,22,25,49,50,55,56,58,60,61,62\}$ \\
144 & $\{36\}$ \\
162 & $\{57\}$ \\
6,552 & $\{63\}$ \\
176,904 & $\{65\}$ \\
\hline
\end{tabular}

Table 3 Two self-dual $[36,18,9]$ codes over $G F(3)$ from the SH matrices of order 36

\begin{tabular}{llc}
\hline $\begin{array}{l}\text { Skew Hadamard } \\
\text { matrices [9] }\end{array}$ & Weight enumerator & $\mid$ PAut(C)| \\
\hline$\{15\}$ & $1+208 y^{9}+40968 y^{12}+1407744 y^{15}+\cdots$ & 8 \\
$\{16\}$ & $1+544 y^{9}+37944 y^{12}+1419840 y^{15}+\cdots$ & 8 \\
\hline
\end{tabular}

\section{$4.7 n=9$}

There are $\geq 18 \mathrm{SH}$ matrices of order 36 [9]. Using the file in [9], the 15th and 16th matrices with construction $C(A)$ produce two inequivalent $[36,18,9]$ ternary self-dual codes (see Table 3). On the other hand, the rest of the matrices with construction $C(A)$ produce 16 inequivalent $[36,18,6]$ ternary self-dual codes. Other constructions $D(A), C\left(A^{T}\right)$, and $D\left(A^{T}\right)$ produce the same set of codes as $C(A)$. We mention that there is only one known ternary self-dual code of length 36 with $d=12$, called the Pless symmetry code.

$4.8 n=10$

There are $\geq 22 \mathrm{SH}$ matrices of order 40 [9]. Over $G F(2)$, construction $C(A)$ produces exactly eight inequivalent extremal Type II binary $[40,20,8]$ codes while the rest are Type II [40, 20,4] codes. Similarly, over $G F(5)$ we obtain one [40, 20, 11] self-dual code, 12 [40, 20, $10]$ self-dual codes, and nine [40, 20,8] self-dual codes. All of these codes over $G F(5)$ are inequivalent. See Table 4 for detail, where the third column follows the index of the matrices in [9], and 12( $\cong 2)$ (similarly for two others) means that the corresponding binary codes are equivalent. We remark that the eight binary Type II codes in the third row of Table 4 have automorphism group orders $64,1,768,32768,768,12,1536,8$, respectively.

We note that the quadratic double circulant (self-dual) code of length 40 over $G F(5)$ has the largest known minimum distance 13 [3], which is, therefore, two larger than the above best code.

\section{$4.9 n=11$}

There are $\geq 59 \mathrm{SH}$ matrices of order 44 [9]. We consider self-dual codes over $G F(11)$ by construction $C(A)$. More precisely, we get exactly 9 [44, 22, 14] self-dual codes, 37 [44, 22, 13] 
Table 4 Self-dual $[40,20]$ codes from the $22 \mathrm{SH}$ matrices of order 40

\begin{tabular}{lll}
\hline Over $G F(q)$ & Minimum distance $d$ & Skew Hadamard matrices [9] \\
\hline$q=2$ & $d=4$ & $\{2,3,4,6,8,9,12(\cong 2), 14(\cong 6), 15,16(\cong 6), 18,19,21,22\}$ \\
$q=5$ & $d=8$ & $\{1,5,7,10,11,13,17,20\}$ \\
& $d=8$ & $\{5,6,7,9,13,14,15,20,22\}$ \\
& $d=10$ & $\{1,2,4,8,10,11,12,16,17,18,19,21\}$ \\
& $d=11$ & $\{3\}$ \\
\hline
\end{tabular}

Table 5 Self-dual [44, 22] codes over $G F(11)$ from the $59 \mathrm{SH}$ matrices of order 44

\begin{tabular}{ll}
\hline Minimum distance $d$ & Skew Hadamard matrices [9] \\
\hline$d=11$ & $\{9,12,39\}$ \\
$d=12$ & $\{6,8,11,13,14,23,35,36,42,49\}$ \\
$d=13$ & The rest \\
$d=14$ & $\{1,5,18,19,28,29,44,51,56\}$ \\
\hline
\end{tabular}

self-dual codes, 10 [44, 22, 12] self-dual codes, and 3 [44, 22, 11] self-dual codes. See Table 5 for detail.

\section{$4.10 n=12$ or 13}

The PH matrix of order 48 is the only known SH matrix of that order [9]. Since 2 and 3 are quadratic residues modulo $47, C(A)$ are quadratic residue codes over $G F(2)$ or $G F(3)$ by Proposition 3.

Let us consider $n=13$. There are $\geq 561 \mathrm{SH}$ matrices of order 52. The first SH matrix of order 52 in [9] gives a self-dual $[52,26,16]$ code over $G F(13)$. This minimum distance is somewhat high. We do not compare this with other possible codes since few self-dual codes over $G F(13)$ are known. We stop considering remaining matrices due to a computational complexity.

\section{$4.11 n=14$}

In [8] $75 \mathrm{SH}$ matrices of order 56 are given. We consider self-dual codes over $G F(2)$ and $G F(7)$. Interesting codes are obtained. In particular, we have checked that there are exactly five extremal Type II $[56,28,12]$ codes from the $75 \mathrm{SH}$ matrices and that only three of the five are inequivalent and they have group orders $24,168,168$, respectively. It is known that there are 16 Type II [56, 26, 12] codes with automorphism of order 13 [20]. So our codes are inequivalent to these codes. Later, Harada [6] constructed at least 1135 Type II $[56,26,12]$ codes from self-orthogonal $3-(56,12,65)$ designs. It will be interesting to check the equivalence of our codes with his codes.

On the other hand, there are self-dual codes over $G F(7)$ with minimum distance $d$ from 10 to 16 . The detail is given in Table 6. There is only one known (quadratic double circulant) self-dual code, denoted by $C_{7,56}$, over $G F(7)$ with minimum distance $d=16[3,4]$. We have checked by Magma that the six nonequivalent codes with $d=16$ in Table 6 are not equivalent to $C_{7,56}$. We observe that none of these six codes obtained in length $56=1+55$ is the extended quadratic residue code of length 56 over $G F(7)$.

Since 7 (resp. 2) is not a square (mod 55), the 75 self-dual codes from SH matrices over $G F(7)$ (resp. $G F(2)$ ) are not even-like duadic codes. 
Table 6 Self-dual $[56,28]$ codes from the 75 SH matrices of order 56

\begin{tabular}{|c|c|c|}
\hline Over $G F(q)$ & Minimum distance $d$ & Skew Hadamard matrices [9] \\
\hline$q=2$ & $\begin{array}{l}d=4 \\
d=8\end{array}$ & $\begin{array}{l}\text { The rest } \\
\{2,3,10(\cong 2), 12,17,21,24(\cong 12), 25,26(\cong 17), 37(\cong 3), \\
40(\cong 3), 47(\cong 17), 50,51,53,54,55,56(\cong 55), 57(\cong 53), \\
58,59,61(\cong 54), 62(\cong 53), 63(\cong 55), 64(\cong 54), 68,69, \\
70,71,72(\cong 50), 73(\cong 51), 74(\cong 58), 75(\cong 59)\}\end{array}$ \\
\hline$q=7$ & $\begin{array}{l}d=12 \\
d=10 \\
d=11 \\
d=12 \\
d=13 \\
d=14 \\
d=15 \\
d=16\end{array}$ & $\begin{array}{l}\{52,60(\cong 52), 65,66(\cong 52), 67\} \\
\{2,3,48,49\} \\
\{1,4,5,24\} \\
\{6,40,47,63,66,72\} \\
\{9,10,12,13,15,17,20,21,23,25,26,29,30,33,37,38\} \\
\{11,14,16,42,45,53,57,58,65,72,73,75\} \\
\text { The rest } \\
\{18,22,31,36,62,68\}\end{array}$ \\
\hline
\end{tabular}

Table 7 Self-dual $[60,30]$ codes from the 22 SH matrices of order 60

\begin{tabular}{lll}
\hline Over $G F(q)$ & Minimum distance $d$ & Skew Hadamard matrices [8] \\
\hline$q=3$ & $d=9$ & $\{1,5,7,13,14,16,18,19\}$ \\
$q=5$ & $d=12$ & $\{2,3,4,6,8,9,10,11,12,15,17,20,21,22\}$ \\
& $d=10$ & $\{8\}$ \\
$d=12$ & $\{12,17\}$ \\
& $d=13$ & $\{13,14,15,16\}$ \\
& $d=14$ & $\{1,2,3,4,5,10,11,21\}$ \\
& $d=15$ & $\{6,7,9,18,19,20,22\}$
\end{tabular}

As a summary, we have the following.

Proposition 6 1. There are exactly five extremal Type II [56, 28, 12] codes from the known 75 SH matrices of order 56, three of which are not equivalent to each other.

2. There exist at least seven inequivalent $[56,28,16]$ self-dual codes over $G F(7)$, six of which are from $\mathrm{SH}$ matrices of order 56.

\section{$4.12 n=15$}

There are $\geq 22$ SH matrices of order 60 [8]. Since $15=3 \cdot 5$, we have self-dual codes over $G F(3)$ and $G F(5)$. More precisely, we obtain 14 ternary self-dual $[60,30,12]$ codes and eight ternary self-dual $[60,30,9]$ codes. We have checked that the 22 codes are all inequivalent. We remark that the best known two ternary self-dual codes of length 60 have minimum distance 18 [4]. Over $G F(5)$ we obtain seven self-dual [60, 30, 15] codes, eight self-dual $[60,30,14]$ codes, four self-dual $[60,30,13]$ codes, two self-dual $[60,30,12]$ codes, and one self-dual $[60,30,10]$ codes. The best known two self-dual codes over $G F(5)$ of length 60 is 18 [4] (see Table 7).

\section{$4.13 n=18$}

There are at least $\geq 90 \mathrm{SH}$ matrices of order 72 [8]. We have checked that these produce Type II codes with minimum distance $d=4,8$, and 12 . In fact, we have plenty of Type II $[72,36,12]$ codes with distinct weight enumerators. More detail will be added $d=16$ is 
open for Type II codes of length 72 . We also have Type III codes with minimum distances in the set $\{9,12,15\}$. The extended quadratic residue code $X Q_{71}$ over $G F(3)$ has $d=18$, and this is the only known code.

$4.14 n=21$

There are at least $\geq 720 \mathrm{SH}$ (non-PH) matrices of order 84 [9]. We obtain Type III codes with minimum distances in the set $\{9,15,18\}$. The optimal distance (obtained for $Q R_{83}$ ) is 21 .

\section{Conclusion and open problems}

In this paper, we have given a coding theoretic proof of Michael's result [11] that the $p$-rank of a skew Hadamard design $\mathbf{D}$ of parameters $(4 n-1,2 n-1, n-1)$ over a field of characteristic $p$ where $p \mid n$ is $2 n$. We thus have shown that the extension of the corresponding incidence matrix produces self-dual codes over $G F(p)$. We also have classified self-dual codes from skew Hadamard matrices of order $4 n(2 \leq n \leq 7)$ and have enumerated self-dual codes from skew Hadamard matrices of order $4 n(8 \leq n \leq 15, n=18,21)$. In particular, we have rediscovered an optimal self-dual $[20,10,8]$ code over $G F(5)$ and discovered six new optimal self-dual $[56,28,16]$ codes over $G F(7)$.

We list some interesting problems for future work as follows.

1. Study self-dual codes over rings, in particular, over $\mathbb{Z}_{4}$ from SH matrices.

2. Give exhaustive lists of SH matrices for $n=8$ and $n=16$.

3. Is there a square Root bound for self-dual codes from SH matrices?

4. Are there Abelian codes from SH matrices?

Acknowledgement Both authors are thankful to M. Harada for pointing out reference [19], to T. S. Michael for providing reference [12] and to E. Spence for explaining his paper [18]. The first author acknowledges partial support by a Project Completion Grant from the University of Louisville.

Open Access This article is distributed under the terms of the Creative Commons Attribution Noncommercial License which permits any noncommercial use, distribution, and reproduction in any medium, provided the original author(s) and source are credited.

\section{References}

1. Assmus E.F. Jr., Key J.D.: Hadamard matrices and their designs: a coding-theoretic approach. Trans. Am. Math. Soc. 330(1), 269-293 (1992).

2. Assmus E.F. Jr., Key J.D.: Designs and Their Codes. Cambridge University Press, Cambridge (1992).

3. Gaborit P.: Quadratic double circulant codes over fields. J. Comb. Theory A. 97, 85-107 (2002).

4. Gaborit P.: http://www.unilim.fr/pages_perso/philippe.gaborit/SD/. Accessed on 9 Oct 2007.

5. Hanaki A., Miyamoto I.: http://kissme.shinshu-u.ac.jp/as/. Accessed on 9 Oct 2007.

6. Harada M.: Self-orthogonal 3- $(56,12,65)$ designs and extremal doubly-even self-dual codes of length 56. Des. Codes Cryptogr. 38, 5-16 (2006).

7. Huffman W.C., Pless V.S.: Fundamentals of Error-Correcting Codes. Cambridge University Press, Cambridge (2003).

8. Kotsireas I.: http://www.medicis.polytechnique.fr/ kotsirea/. Accessed on 9 Oct 2007.

9. Koukouvinos C.: http://www.math.ntua.gr/people/ckoukouv/hadamard.htm. Accessed on 9 Oct 2007.

10. MacWilliams F.J., Sloane N.J.A.: The Theory of Error Correcting Codes. North Holland, Amsterdam (1981).

11. Michael T.S.: The p-ranks of skew Hadamard designs. J. Comb. Theory Ser. A 73, 170-171 (1996).

12. Mirsky L.: An Introduction to Linear Algebra. Dover Edition, New York (1990). 
13. Pless V.: Duadic codes and generalizations. Eurocode'92 (Udine, 1992), 3-15, CISM Courses and Lectures, 339, Springer, Vienna (1993).

14. Pless V., Sloane N.J.A.: On the classification and enumeration of self-dual codes. J. Comb. Theory A 18, 313-335 (1975).

15. Rushanan J.J.: Duadic codes and difference sets. J. Comb. Theory Ser. A 57, 254-261 (1991).

16. Seberry J., Yamada M.: Hadamard matrices, sequences, and block designs. In: Dinitz J.H., Stinson D.R. (eds.) Contemporary Design Theory: A Collection of Surveys, pp. 431-560. John Wiley, New York (1992).

17. Spence E.: http://www.maths.gla.ac.uk/ es/hadamard.html. Accessed on 9 Oct 2007.

18. Spence E.: Classification of Hadamard matrices of order 24 and 28. Discrete Math. 140(1-3), 185-243 (1995).

19. Wakabayashi T.: On the self-dual codes over GF(7) generated by Hadamard matrices of order 28 . AKCE Int. J. Graphs Comb. 1(1), 41-49 (2004).

20. Yorgov V.: A method for constructing inequivalent self-dual codes with applications to length 56. IEEE Trans. Inform. Theory 33, 72-82 (1987). 